\title{
Design of Distance Education Business for Wireless Broadcasting Networks
}

\author{
Shimin Liu ${ }^{1}$, Lingjun Yang ${ }^{2}$ \\ Information Engineering School, \\ Communication University of China \\ Beijing, China \\ liushimin28@163.com
}

\author{
Sanxing $\mathrm{Cao}^{3,}$ \\ New Media Institute, \\ Communication University of China, \\ Beijing, China
}

\begin{abstract}
With the rapid development of Internet,the system of Radio Film and Television has faced a grave challenge,Over The Top(OTT) TV emerges as the times required. So in this paper,a platform of distance education based on Browser/Server is designed.Apache +PHP + MySQL are used in this platform, and Ueditor are introduced in edit module.Teacher edit lessons by Ueditor,and release them through the system.Users can watch lessons on TV terminal finally. It changes the one-way transmission,and meets audience's demands for the trend to convergence of traditional media and new media.
\end{abstract}

\section{Keywords-Distance Education Business;PHP Template;Rich} Text Editor

\section{INTRODUCTION}

In the background of media convergence and technology innovation,radio and television agencies are supposed to make a breakthrough in the broadcast model which is lack of interactivity and single business, and introduce the interactive system into infrastructure including wireless broadcasting networks.In this instance,whether they could develop and apply interactive business rapidly,operate and administrate services has became the key factor to new media industry development[1].On account of this situation, a digital platform especially integrated business base on set-top box are very suitable to meet the market demands that mainly refer to interactivity.

A platform of distance education for wireless broadcasting networks was designed in this paper,users can learn a large number lessons on TV screen which edited by administrator through Ueditor,text,picture,radio and video included.After learning, users could grade the lesson, and administrator will adjust the contents according to the mark given by users. The system develop base on Apache+PHP+MySQL, witch is free to build a network platform, and PHP is open source,the system will be easy to program and debug.Apache,PHP and MySQL can link to each other closely,and they are convenient and flexible for programmer's operation,Linux, Unix and Windows all could be as the operating system for both of them[2].

\section{DESIGN OF Distance EdUCATION BUSINESS}

\section{A. Distance education system framework}

The distance education system based on Browser/Server, which simplified the development,maintenance and use of software by unifying the client side and concentrating the core part of function realization on the server,Apache +PHP + MySQL are used in this platform,and they includes three modules:edit module ,server module and display module,as shown in Fig.1.

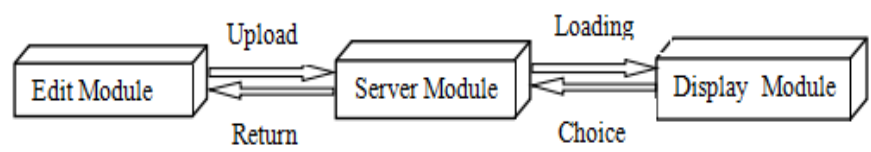

Fig. 1. Distance Education Framework

In edit module,PHP+MySQL were used to implement data access, $\mathrm{PHP}+\mathrm{MySQL}$ are great combination to operate database.Before upload to the server,we need to transform dynamic web page into static page, template can be used there.This template can reduce the burden on the server,and improve system's readability and maintainability.In order to present diversity style in display module,HTML+CSS+JavaScript were used to achieve it.HTML+CSS+JavaScript is a main current development pattern of Web application.HTML is a markup language to structure and semanticize information,Cascading Style Sheets(CSS) add diversified styles to structuring document,while JavaScript can respond user's action[3].

\section{B. Design of self-adaption about style}

The style of this business is designed on computer,while it also need to present on TV screen. Resolution of them are different,so we are supposed to design self-adaptive to make it present correctly.Display terminal width divide by design' $\mathrm{s}$ width is the scaling that is needed in next arithmetic, 
marked \$scale.For left and top, multiply by \$scale and add to relevant value of display terminal,that is suitable for terminal [4]. The relevant codes are shown below:

1. \$scale =\$canvas["width"]/\$canvas_width;

\section{2. foreach (\$json as $\$ v)\{$}

3. $\$ v->$ css- $>$ width $=$ floor(intval $(\$ v->c s s->$ width $) * \$ s c a l e)$;

4. $\$ v$->CSs-> height=height(intval( $\$ v$ - >css- $>$ height $) * \$ s c a l e)$;

5. if (isset $(\$ v->$ css- $>$ left $))\{$

6. $\$ v->$ css- $>$ left $=$ floot $($ intval $(\$ v->$ css- $>$ left $) * \$$ scale +

7. \$canvas['left']);

8. \} else \{

9. $\$ v->c s s->l e f t=\$ c a n v a s[' l e f t ']$;

10. $\}$

11. if (isset $(\$ v->$ css- $>$ top $))\{$

12. $\$ v->$ css- $>$ top $=$ floot $($ intval $(\$ v->$ css $->$ top $) * \$$ scale +

13. \$canvas['top']);

14. \} else \{

15. $\$ v->$ css- $>$ top $=\$$ canvas $\left[{ }^{\prime} t o p '\right]$;

16. $\}$

In addition, the value of border, width, height,left and top is supposed to amend,.On the one hand, a default value could be given for attribute that have not given before.On the other hand,it can avoid frame exceeding the area owning to tiny calculation error.

\section{Apply the System for users and administrator}

Administrator need to login in the system in design terminal,and get authority to access to main interface.Then administrator have permission to add,delete and modify the courses.For example, administrators can adjust course's order according to page views and user's feedback,publish new course compiled by Ueditor and save into database, and delete redundant or out of time lessons. The process is shown as Fig.2

In the system, the set-top box's function is same as browser, it make television get information from Internet instead of traditional signal,and changes the one-way transmission, and meets audience's demands for the trend to convergence of traditional media and new media.

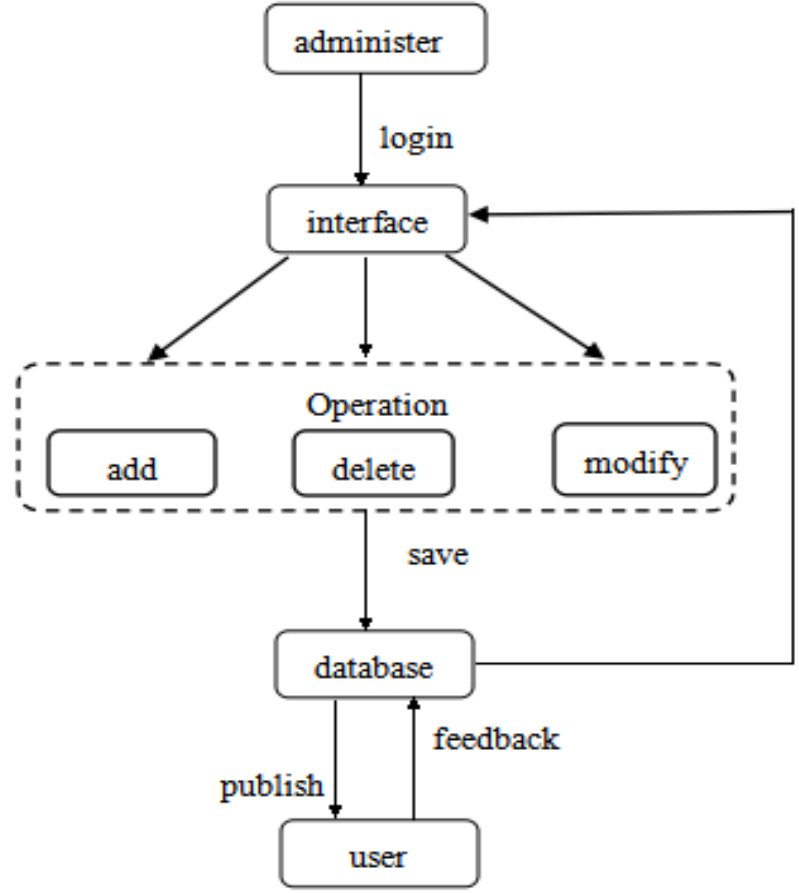

Fig. 2. The Process of Distance Education Business

\section{TeChnology Used In System}

\section{A. Rich Text Editor}

It is especially convenient to edit courses by Ueditor,a rich text editor researched and developed by Baidu company. The rich text editor can provide text editing function similar to Microsoft Word.For compatibility,although there is still lacking of criterion for rich text editor,all browsers have unify application program interface(API) for basic editing function.Users can download the editor in Internet,and embed in web page,then they edit text style,upload picture, video and table[5].The advantage of Ueditor is it allows us to modify their codes, provide visual function selection and customized document.Developers can choose various source codes base on all kinds of programming language. When use it,we just need add it to the page and modify the path in ueditor.config.js, the relevant codes is shown below :

\section{\#Call the script}

1. <script type="text/javascript" $>$

2. var editor = new UE.ui.Editor();

3. editor.render("myEditor");

// editor instantiation

4. $</$ script $>$ 
\# Set Path in ueditor.config.js

\section{1. function ()\{}

2. $\operatorname{var} U R L=" / e d u /$ ueditor/";

// the relative path to the root directory of the web site

\section{3. getUEBasePath();}

\section{B. PHP Template and Smarty}

The PHP template separate code development from page design,thus enhance readability of date about PHP, and simplify maintenance and update of website. It is a major technology to alleviate server's burden[6].For dynamic pages,each time users require to server is a process to call PHP script. If we can cache the script execution or put it into a HTML page, users can visit the static pages directly rather than dynamic pages. To generate static pages by template, a blank template page without any content is needed at first,then replace by relevant content got from database .Finally,All of the content will be written into a new HTML document, that is the static page what we need.The process is shown as Fig.3.

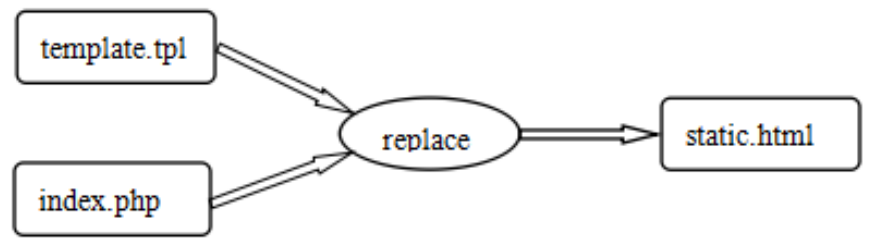

Fig. 3. The Process of Template Engine

There are many kinds of template engines, while Smarty is one of the most famous one.Compared to other templates, Smarty has following advantages.

- Fast speed:the program using Smarty is faster than others.

- The compiled template:Smarty is a kind of compiled template engines, it will compile the program with Smarty into PHP files are comprised of PHP code and HTML.If the source program is unchanged,when access to program next time,it will open the compiled file.

- Cache technology:Smarty use this technology that can transform the final present page into static HTML.User can call the cache file next directly rather than call the template.

- The plug-in technology:Users can customize the plugin by themselves in Smarty.

So in this system,Smarty is used to generate static pages.One thing require to noted is that in login part we are not supposed to use Smarty in consideration of security.

\section{CONCLUSION}

A system about distance education business is designed and implemented in this paper,administrators can publish course in design terminal ,and transmit to display terminal.Users can choose them by themselves on TV terminal, interactive is it's most advantage.In next part,we will add to payment to charge for some businesses[7].Because of the development of Internet and the lower price of broadband,varieties of interactive businesses is a main desire for the cable operator,and distance education is one of the most important business of that.As a result,the business for Wireless Broadcasting Networks will get a great profit in the future.

\section{ACKNOWLEDGMENT}

This work is sponsored by the 382 Talents Plan (G08382304), Chinese National Hi-tech Industrialization Project granted by China Art Science and Technology (CNGI09-03-15-1), and the Virtual Library Technology Institute.

\section{References}

[1] Tang Baihui,Cao Sanxing:Design of the Integrated Business Supporting Platform of Wireless Broadcasting Networks[A].2011 International Coverageand Transmission Conference.Xi'an,China.2011.pp. 567-570.

[2] Feng Xingli,Suo Zhihua,Xu Mo.Security and Website Static Technology of WEB System Based on PHP+MySQL[J].Modern Electronics Technique,2012,08:25-27.

[3] Ma Liming.A Study of the Designing and Rendering Technology for the Multi-screen Application under the Architecture of Omni Media [D]. Communication University of China,2014.

[4] Xia Zhiqiang.Design and Implementation on Rendering and Extending for Interactive Service Based on Set-top Boxes [D].Communication University of China,2014.

[5] Wang Jun, Web-Based Learning Resource Online Editor Research and Development[D].Beijing University of Posts and Telecommunications, 2012

[6] Zhong Linlin,Wang Mo.Learning PHP From Entry to Master[M].Beijing:Chinese railway press,2014:362.

[7] Jin Jing. Design of Omnimediad Distance Education Business of Platform[J].Electronic Technology and Software Engineering,2013,17:17-19. 